EPJ Web of Conferences 59, 03005 (2013)

DOI: $10.1051 /$ epjconf/20135903005

(C) Owned by the authors, published by EDP Sciences, 2013

\title{
Analytical criterion for shock ignition of fusion reaction in hot spot
}

\author{
X. Ribeyre ${ }^{\text {, }}$ V.T. Tikhonchuk, J. Breil, M. Lafon, A. Vallet and E. Le Bel \\ University Bordeaux - CNRS - CEA, Centre Lasers Intenses et Applications, UMR 5107, \\ 33405 Talence, France
}

\begin{abstract}
Shock ignition of DT capsules involves two major steps. First, the fuel is assembled by means of a low velocity conventional implosion. At stagnation, the central core has a temperature lower than the one needed for ignition. Then a second, strong spherical converging shock, launched from a high intensity laser spike, arrives to the core. This shock crosses the core, rebounds at the target center and increases the central pressure to the ignition conditions. In this work we consider this latter phase by using the Guderley self-similar solution for converging flows. Our model accounts for the fusion reaction energy deposition, thermal and radiation losses thus describing the basic physics of hot spot ignition. The ignition criterion derived from the analytical model is successfully compared with full scale hydrodynamic simulations.
\end{abstract}

\section{INTRODUCTION}

Inertial confinement fusion relies on the central hot spot ignition following the implosion of a spherical capsule by the laser ablation pressure. The ignition conditions are achieved by a partial conversion of the shell kinetic energy into the internal energy of a hot spot. The simulations show that such ignition conditions in the direct or indirect drive scheme can be achieved for the shell implosion velocity of $350-400 \mathrm{~km} / \mathrm{s}$. The hydrodynamic instabilities of the imploding shell are identified as a serious risk factor.

A safer, more stable ignition can be achieved by separation of the compression and ignition steps. The compression can be accomplished at a lower implosion velocity of $\sim 200-300 \mathrm{~km} / \mathrm{s}$, and the fuel in the hot spot is ignited by launching a strong spherical converging shock before the shell stagnation [1-3]. The ignition shock launched from the outside imploding shell makes a complicated evolution before reaching the central hot spot. First, it converges and crosses the return shock in the shell. It is amplified in this collision and enters in the compressed region just before the stagnation time [5, 7]. Then, it enters in the hot spot, converges to the center, and heats the core after the rebound. At this last stage the hot spot fuel is supposed to ignite.

We propose in this paper a model that describes this latter phase of the ignition process, when a strong shock penetrates inside the DT core. It is based on the self-similar solution of a convergingdiverging spherical shock obtained by Guderley [4]. The model accounts for the fusion reactions and the thermal and radiation energy losses from the core. The predicted ignition conditions are in good agreement with full scale numerical simulations.

\footnotetext{
ae-mail: ribeyre@celia.u-bordeaux1.fr
}

This is an Open Access article distributed under the terms of the Creative Commons Attribution License 2.0, which permits unrestricted use, distribution, and reproduction in any medium, provided the original work is properly cited. 


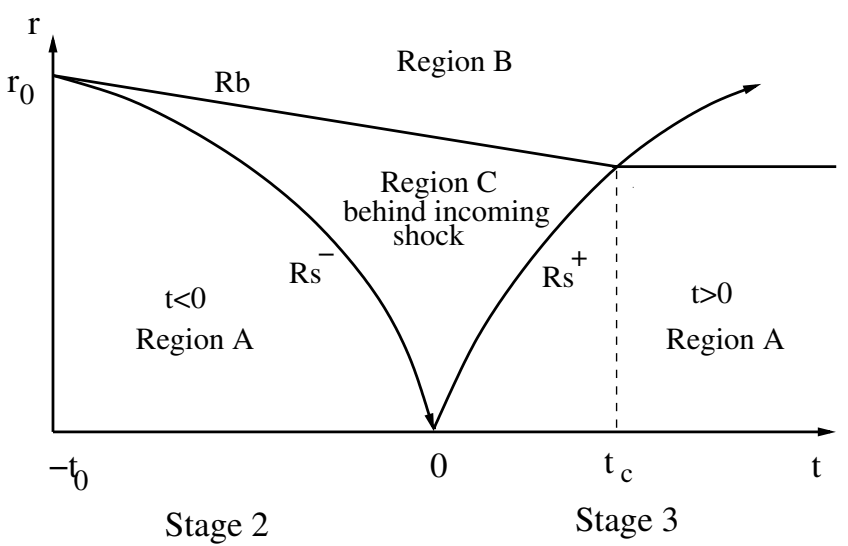

Figure 1. Trajectories of the ingoing $\left(R_{s}^{-}\right)$and outgoing $\left(R_{s}^{+}\right)$shocks in the core and the position of the inner surface of the shell $R_{b}$.

\section{SELF-SIMILAR SOLUTION}

Our analytic model considers the central part of fuel inside the cold dense shell, which acts as a piston. It launches a spherical converging shock inside the central region (A) presented in Fig. 1. The domain of the flow is delimited by the shell position $R_{b}(t)$, while the shock front coordinate is $R_{s}^{-}$for the converging part and $R_{s}^{+}-$for the diverging part. The core initial radius is $r_{0}$ at the time $t=-t_{0}$, and the time $t=0$ corresponds to the shock rebound from the center. The reflected shock propagates outward, compresses and heats the fuel thus creating the hot spot. The ignition conditions are assumed to be achieved at the moment $t_{c}$ when the reflected shock enters the shell.

The hydrodynamic flow behind the shock propagating through a homogeneous fluid is described by the self-similar solution [4]. The initial pressure and temperature are assumed to be negligibly small compared to the values behind the shock. Although the conditions of validity of this solution are not exactly fulfilled in the real target, it describes reasonably well the ignition criterion. This is explained by the fact that the shock amplitude increases rapidly as it converges to the center, so that the initial pressure has little effect on the conditions behind the front.

The fuel is considered as a fully ionized ideal DT gas with the polytropic coefficient $\gamma=5 / 3$. The core is described by the equations of ideal hydrodynamics in spherical geometry for the density $\rho$ and the fluid velocity $u$ :

$$
\begin{aligned}
& \partial_{t} \rho+\partial_{r}(\rho u)+2 \rho u / r=0, \quad \partial_{t} u+u \partial_{r} u+\left(c_{s}^{2} / \gamma \rho\right) \partial_{r} \rho+\left(2 c_{s} / \gamma\right) \partial_{r} c_{s}=0, \\
& \left(\partial_{t}+u \partial_{r}\right)\left(c_{s}^{2} / \rho^{\gamma-1}\right)=0
\end{aligned}
$$

where $c_{s}=(\gamma p / \rho)^{1 / 2}$ is the acoustic velocity and the pressure $p=p_{0}\left(\rho / \rho_{0}\right)^{\gamma}$ is given by the equation of state of an ideal gas. The self-similar solution of these equations for a converging shock can be presented in the following form [4]:

$$
u(r, t)=(\alpha r / t) U(\xi), \quad c_{s}(r, t)=(\alpha r / t) C(\xi), \quad \rho(r, t)=\rho_{0} G(\xi),
$$

where $\xi=\left(r / r_{0}\right) /\left|t / t_{0}\right|^{\alpha}$ is the self-similar coordinate and $\alpha$ is a free parameter. The ordinary differential equations for the scaling functions $G, U$ and $C$ can be found elsewhere [7]. The value for $\alpha=0.688377$ is determined numerically. The radial profiles of density and pressure are plotted in Fig. 2 before and after the collapse time. The time moment $0.32 t_{0}$ corresponds to the shock entering in the piston. 

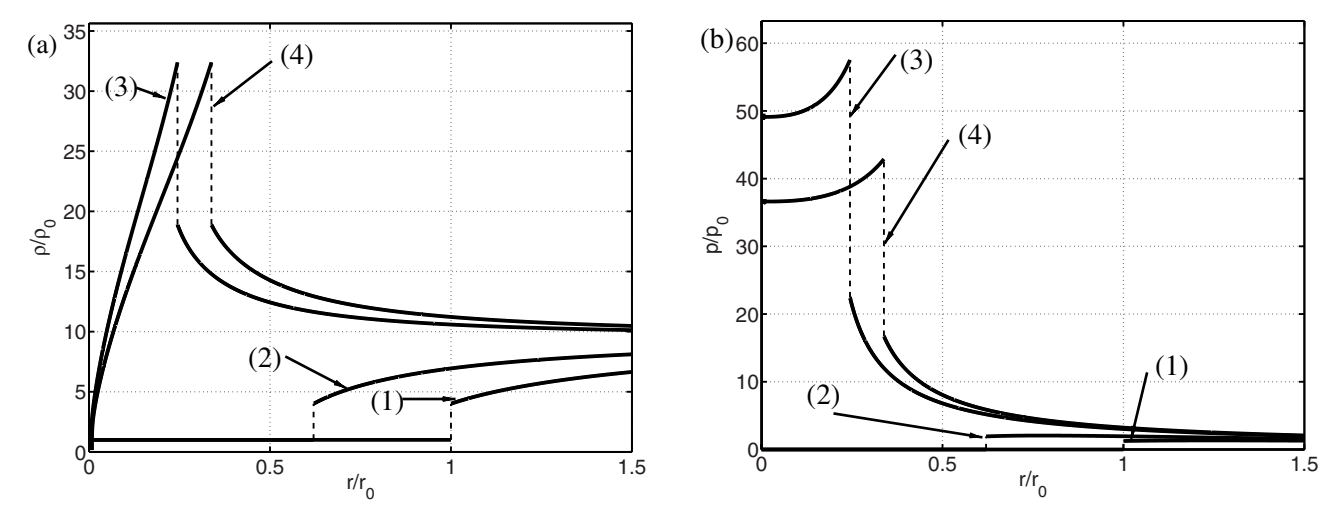

Figure 2. (a) Density and (b) pressure radial profiles at the time moments: $t / t_{0}=-1$ (1), $t / t_{0}=-0.5$ (2), $t / t_{0}=0.25(3)$, and $t_{c} / t_{0}=0.32$ (4).

The initial temperature $T_{0}=m_{i} u_{0}^{2} / 2 \gamma$ and pressure $p_{0}=\rho_{0} u_{0}^{2} / \gamma$ are defined by the initial fuel density, $\rho_{0}$ and the shock velocity $u_{0}$. Here, $m_{i}=\left(m_{D}+m_{T}\right) / 2$ is the average ion mass.

Before the shock collapse, the density is increased by a factor 4 behind the shock front. The increments in density and pressure are relatively modest in the converging shock. In contrast, in the diverging shock, the density shows a triangular shape with the maximum compression of 32.39; the pressure amplification is 2.56 , and it remains approximately constant behind the front. The central pressure is increased by a factor of 40 at the exit time $t=t_{c}$. Such a pressure configuration is strongly non-isobaric as it is expected in the shock ignition scheme $[2,3]$.

\section{FUEL IGNITION CONDITIONS}

Assuming that the dissipation processes do not alter strongly the hydrodynamic flow, this self-similar solution could be applied for obtaining the shock ignition criteria. As initial conditions, we consider the density of the hot spot $\rho_{0}$ at stagnation and the shock front velocity $u_{0}$ at the moment when it penetrates inside a pre-compressed hot spot. Firstly, we calculate the energy needed to launch this shock and compress the deuterium-tritium (DT) fuel. The ignition conditions are evaluated in function of these two parameters and the hot spot radius $r_{0}$ that defines the collapse time $t_{0}=\alpha r_{0} /\left|u_{0}\right|$. As the major energy is brought to the fuel after the shock collapse, we estimate the fusion energy balance, the radiative and thermal conduction losses at the shock exit time $t_{c}=0.324 t_{0}$.

The power delivered by the piston to the shock is given by the pressure $p_{b}$ and the fluid velocity $u_{b}$ at the external surface of the radius $R_{b}(t)$, see Fig. 1: $d_{t} E_{s}=-4 \pi R_{b}^{2} p_{b} u_{b}$. The total shock energy is calculated by integrating the power $d_{t} E_{s}$ over the time from $-t_{0}$ to $t_{c}=0.324 t_{0}$ :

$$
E_{s}=A_{s}\left(t_{c}\right) \rho_{0} r_{0}^{3} u_{0}^{2}
$$

where $A_{s}\left(t_{c}\right)=5.92$.

In the same way one can estimate the fusion thermonuclear energy released behind the outgoing shock in the time interval $\left(0, t_{c}\right)$. Assuming that the neutrons are leaving the fuel and the $\alpha$ particles are depositing their energy inside, we account only for the released $\alpha$-particle power: $d_{t} E_{f}=\pi f_{\alpha} W_{\alpha} \int_{0}^{R_{s}^{+}} r^{2} \rho^{2}\langle\sigma v\rangle d r / m_{i}^{2}$, where $W_{\alpha}=3.5 \mathrm{MeV}$ is the $\alpha$-particle energy released in DT reaction, $f_{\alpha}$ is the fraction of $\alpha$-particles energy deposited inside the hot-spot, and $\langle\sigma v\rangle$ is the DT reactivity. It can be approximated by a quadratic function $\langle\sigma v\rangle \approx \beta T^{2}$ for the DT temperature varying between 8 and $25 \mathrm{keV}$, where $\beta=8.2 \times 10^{-19} \mathrm{keV}^{-2} \mathrm{~cm}^{3} / \mathrm{s}$. Correspondingly, the fusion power 


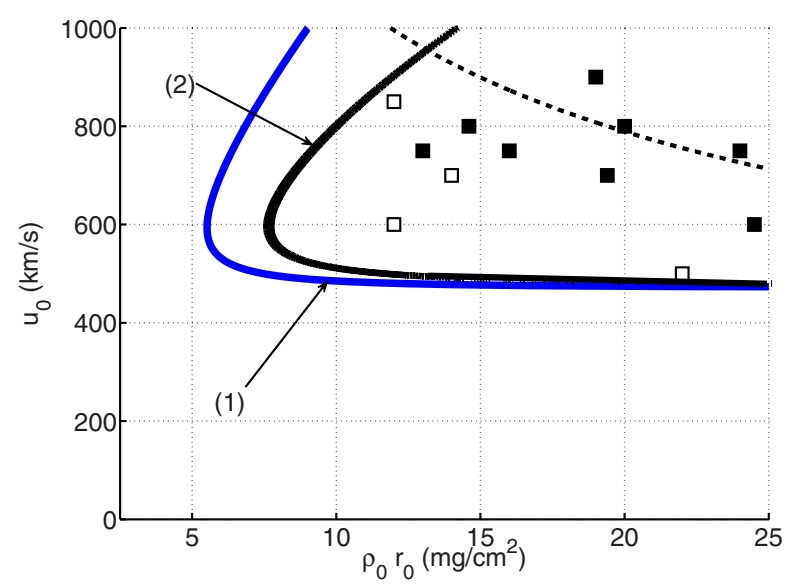

Figure 3. Dependence of the areal density $\rho_{0} r_{0}$ of the fuel assembly on the initial shock velocity. Blue solid curve (1) for total $\alpha$-particle deposition and black solid curve (2) with $\alpha$ fraction. The dashed curve represents the criterion given in Ref. [1]. The square symbols present the results of full numerical simulation: black - ignited targets, white - no ignition.

writes: $d_{t} E_{f}=f_{\alpha} A_{f} \rho_{0}^{2} u_{0}^{4} r_{0}^{3}$ where:

$$
A_{f}(t)=\pi W_{\alpha} \beta(2 \gamma)^{-2} \xi_{s}^{7} G^{2}\left(\xi_{s}\right) C^{4}\left(\xi_{s}\right)\left(t / t_{0}\right)^{7 \alpha-4} / 3 .
$$

As the fusion power increases with time, we take it at the collision time $t_{c}: d_{t} E_{f}=f_{\alpha} A_{f}\left(t_{c}\right) \rho_{0}^{2} u_{0}^{4} r_{0}^{3}$ where $A_{f}\left(t_{c}\right)=8.1 \times 10^{-13} \mathrm{~W}$ with $\rho_{0}$ given in $\mathrm{g} / \mathrm{cc}, r_{0}-$ in $\mathrm{cm}$ and $u_{0}-$ in $\mathrm{cm} / \mathrm{s}$.

The expression for the $\alpha$-particle energy deposition fraction $f_{\alpha}$ [9] depends on the ratio $\tau_{\alpha}$ between the hot spot radius, $R_{h}=R_{s}^{+}=0.34 r_{0}$ and the $\alpha$-particle mean free path, $\lambda_{\alpha}$. The latter can be also expressed through the self-similar solution. Then the energy deposition fraction $f_{\alpha}$ depends only on the initial areal mass density $\rho_{0} r_{0}$ and the initial shock velocity $u_{0}$. The domain of parameters where $f_{\alpha}>0.5$ corresponds to shock velocities below $700-800 \mathrm{~km} / \mathrm{s}$, which are of practical interest.

At the ignition threshold, the released fusion power has to be compared with the radiative and electron losses. The photon range for a typical hot spot temperature of about $4 \mathrm{keV}$ and density $30 \mathrm{~g} / \mathrm{cc}$ is more than $60 \mathrm{~g} / \mathrm{cm}^{2}$, which is much larger than the areal density of the hot spot. Then, neglecting the re-absorption of photon, the radiation power loss reads: $d_{t} E_{r}=4 \pi A_{b} \int_{0}^{R_{s}^{+}} r^{2} \rho^{2} T^{1 / 2} d r$ where $A_{b}=2.8 \times 10^{16} \mathrm{~W} \mathrm{~cm}^{3} \mathrm{~g}^{-2} \mathrm{keV}^{-1 / 2}$. Accomplishing the integration over the radius, we present the radiation power in the form: $d_{t} E_{r}=A_{r}(t) \rho_{0}^{2} u_{0} r_{0}^{3}$ where

$$
A_{r}(t)=\frac{4}{3} \pi A_{b} \xi_{s}^{4} G^{2}\left(\xi_{s}\right) C\left(\xi_{s}\right)\left(t / t_{0}\right)^{4 \alpha-1}\left(2 \gamma / m_{i}\right)^{-1 / 2} .
$$

The value of the coefficient $A_{r}$ at the collision time $t_{c}$ is $A_{r}\left(t_{c}\right)=1.70 \times 10^{11} \mathrm{~W}$.

The particle energy losses are defined by the electron heat flux $q_{e}=-\chi_{e} \nabla T_{e}$ where $\chi_{e}=$ $K_{e} T^{5 / 2} / \ln \Lambda$ is the Spitzer conductivity with $K_{e}=3 \times 10^{13} \mathrm{~W} \mathrm{~cm}^{-1} \mathrm{keV}^{-7 / 2}$ and $\ln \Lambda \simeq 6$ is the Coulomb logarithm. By calculating the temperature gradient on the shock wave front, the thermal conduction power losses, $d_{t} E_{e}=4 \pi R_{s}^{+2} q_{e}$, can be written as $d_{t} E_{e}=A_{e}(t) u_{0}^{7} r_{0}$, where the time dependent coefficient

$$
A_{e}(t)=-8 \pi K_{e}(\ln \Lambda)^{-1}\left(2 \gamma / m_{i}\right)^{-7 / 2}\left(t / t_{0}\right)^{(8 \alpha-7)} \xi_{s}^{8} C^{6}\left(\xi_{s}\right)\left[C\left(\xi_{s}\right)+\xi_{s} C^{\prime}\left(\xi_{s}\right)\right] .
$$

The value of $A_{e}$ at the collision time $t_{c}$ is $A_{e}\left(t_{c}\right)=1.2 \times 10^{-40} \mathrm{~W}$.

Knowing the $\alpha$-particle heating, the radiative and thermal losses, the ignition criterion is evaluated at $t=t_{c}$ in the following form: $d_{t} E_{f}>d_{t} E_{r}+d_{t} E_{e}$. It provides a relation between the threshold areal 
density of the hot spot $\rho_{0} r_{0}$ and the initial shock velocity $u_{0}$ in the implicit form:

$$
\rho_{0} r_{0}=\sqrt{A_{e}\left(t_{c}\right) u_{0}^{6} /\left(f_{\alpha} A_{f}\left(t_{c}\right) u_{0}^{3}-A_{r}\left(t_{c}\right)\right)} .
$$

The curve defined by Eq. (2) is plotted in Fig. 3 for two situations. The blue solid curve (1) shows the ignition condition assuming no $\alpha$-particle energy losses $f_{\alpha}=1$. The black solid curve (2) shows the ignition threshold with the $\alpha$-particle losses taken into account. The minimum shock velocity $u_{0 \min }$ is defined as the ignition condition for very large densities. For $f_{\alpha}=1$ its value follows from Eq. (2): $u_{0 \min }=\left[A_{r}\left(t_{c}\right) / A_{f}\left(t_{c}\right)\right]^{1 / 3} \simeq 470 \mathrm{~km} / \mathrm{s}$. The $\alpha$-particle losses increase this value to $u_{0 \min } \simeq 500 \mathrm{~km} / \mathrm{s}$. This value is determined essentially by the radiation losses and has the same role as the Post temperature in the conventional central hot spot ignition.

The minimum areal density of the assembled fuel,

$$
\left.\rho_{0} r_{0}\right|_{\min } \simeq 10 \mathrm{mg} / \mathrm{cm}^{2}
$$

is achieved at the shock velocity $u_{0} \simeq 600 \mathrm{~km} / \mathrm{s}$.

A criterion for the fuel ignition by a shock was obtained by Shcherbakov [1]. The analytical form of this criterion is written as: $\rho_{0} r_{0} V_{b 0}^{2.2} \geq 1$, where $V_{b 0}=V_{b}\left(t=-t_{0}\right)$ is the mass velocity behind the front of the converging shock in units of $100 \mathrm{~km} / \mathrm{s}$ and the initial areal density is in $\mathrm{g} / \mathrm{cm}^{2}$. A comparison of this formula with our criteria (2) and (3) and numerical simulations (square symbols) shows significant differences. For comparison, we rewrite this expression in terms of the initial shock velocity $u_{0}$. According to the boundary condition $V_{b 0}=0.75 u_{0}$. Then Shcherbakov's criterion $\rho_{0} r_{0} u_{0}^{2.2} \geq 1.87$, is shown by the dashed-curve in Fig. 3. For the initial areal density range $\rho_{0} r_{0}=10-25 \mathrm{mg} / \mathrm{cm}^{2}$, this criterion gives the ignition shock velocity in the range $1000-700 \mathrm{~km} / \mathrm{s}$, which is $\sim 30 \%$ greater than the shock velocity given by Eq. (2) with the $\alpha$-particle losses taken into account. Moreover, a monotonous dependence of the areal density on the shock velocity in Shcherbakov's criterion does not allow to obtain the minimum areal density Eq. (3) or the threshold shock velocity, $u_{0 \mathrm{~min}}$, for ignition.

\section{CONCLUSIONS}

The ignition threshold predicted by the self-similar model is compared in Fig. 3 with the numerical simulations with the radiation hydrodynamics code CHIC [8]. The simulations were conducted in the spherical geometry with the all-DT shell target described in detail in Refs. [3, 5]. The values of the hot spot areal density and the shock front velocity were measured in the simulations and presented in Fig. 3 . The simulations with full physics taken into account give the minimum shock velocity $u_{0} \approx 800 \mathrm{~km} / \mathrm{s}$ and the minimum areal density $\rho_{0} r_{0} \approx 15 \mathrm{mg} / \mathrm{cm}^{2}$, which is $25 \%$ greater than the analytical criteria.

This difference can be attributed to the oversimplifying assumptions of the model. The conditions in the simulations at the moment when the shock enters in the hot spot correspond to a temperature of several $\mathrm{keV}$ and a pressure of $10 \mathrm{Gbar}$. The shock Mach number at this moment is $M \simeq 2$. The value $1 / M \simeq 50 \%$ gives the estimate of the accuracy of the model. Moreover, in our simplified model the energy transport processes are accounted for as perturbations and the fuel density profile at stagnation may affect the results. Nevertheless, in spite of these simplifying assumptions, the model provides rather reasonable agreement with the full hydrodynamical simulations. That proves that it accounts quite well for the basic physics relevant for shock ignition. It describes the pressure amplification corresponding to the non-isobaric fuel configuration and provides reasonable estimates for the ignition conditions.

\section{References}

[1] V. A. Shcherbakov, Sov. J. Plasma Phys. 9, 240 (1983)

[2] R. Betti et al., Phys. Rev. Lett. 98, 155001 (2007)

[3] X. Ribeyre et al., Plasma Phys. Control. Fusion 51, 124030 (2009) 


\section{EPJ Web of Conferences}

[4] V. G. Guderley, Luftfahrforschung 19, 302 (1942)

[5] M. Lafon et al., Phys. Plasmas 17, 052704 (2010)

[6] S. Atzeni and J. Meyer-ter-Vehn, The Physics of Inertial Fusion, pp. 170-190, Oxford University Press, New York (2004)

[7] X. Ribeyre et al., Phys. Plasmas 18, 102702 (2011)

[8] P. H. Maire et al., SIAM J. Sci. Comput. 29, 1781 (2007)

[9] O. N. Krokhin and V. B. Rozanov, Sov. Journ. Quantum Electron. 2, 393 (1973) 